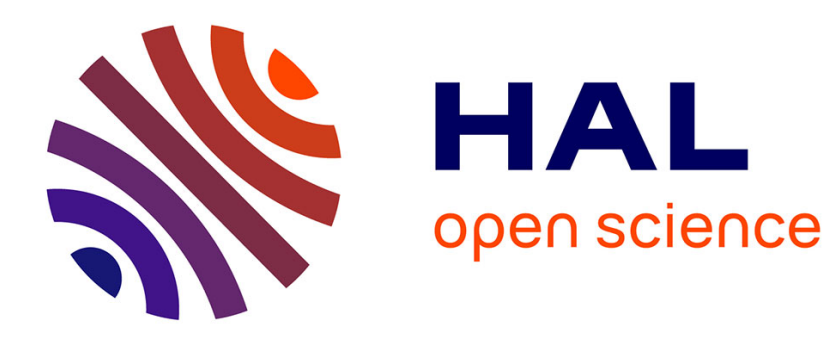

\title{
Quadrupolar glass ordering in solid deuterium
}

N.S. Sullivan, M. Devoret, J.M. Vaissière

\section{To cite this version:}

N.S. Sullivan, M. Devoret, J.M. Vaissière. Quadrupolar glass ordering in solid deuterium. Journal de Physique Lettres, 1979, 40 (21), pp.559-564. 10.1051/jphyslet:019790040021055900 . jpa-00231688

\section{HAL Id: jpa-00231688 https://hal.science/jpa-00231688}

Submitted on 1 Jan 1979

HAL is a multi-disciplinary open access archive for the deposit and dissemination of scientific research documents, whether they are published or not. The documents may come from teaching and research institutions in France or abroad, or from public or private research centers.
L'archive ouverte pluridisciplinaire HAL, est destinée au dépôt et à la diffusion de documents scientifiques de niveau recherche, publiés ou non, émanant des établissements d'enseignement et de recherche français ou étrangers, des laboratoires publics ou privés. 


\title{
Quadrupolar glass ordering in solid deuterium
}

\author{
N. S. Sullivan, M. Devoret and J. M. Vaissière \\ Centre d'Etudes Nucléaires de Saclay, Service de Physique du Solide et de Résonance Magnétique, \\ B.P. no 2, 91190 Gif-sur-Yvette, France
}

(Reçu le 27 juin 1979, accepté le 13 septembre 1979)

\begin{abstract}
Résumé. - Nous avons effectué des mesures des raies d'absorption RMN du deutérium solide afin d'établir le diagramme de phase de l'ordre orientationnel pour les concentrations d'espèces $J=1,42<X<75 \%$, et pour les températures $0,035<T<2,5 \mathrm{~K}$. Comme dans l'hydrogène solide, on observe trois phases : une phase désordonnée à haute température, une phase présentant un ordre à longue distance pour $X>X_{\mathrm{c}} \approx 55 \%$ et pour $X<X_{\mathrm{c}}$ une nouvelle phase encore jamais observée dans le deutérium solide.

La nature de l'ordre dans cette nouvelle phase a été déterminée grâce à l'analyse des formes de raies de résonance. Il y a une distribution large du paramètre d'ordre local pour $X<X_{\mathrm{c}}$ et nous interprétons ce résultat par un verre quadrupolaire analogue à celui observé dans l'hydrogène solide.
\end{abstract}

\begin{abstract}
Measurements of the NMR absorption line shapes have been used to establish the phase diagram for orientational ordering of the $J=1$ molecules in solid mixtures of paradeuterium $(J=1)$ and orthodeuterium $(J=0)$ for para concentrations $42<X<75 \%$ and temperatures $0.035<T<2.5 \mathrm{~K}$. As in solid hydrogen, we observe three phases : a rotationally disordered phase, a long range orientationally ordered phase for concentrations of the $J=1$ species greater than a critical concentration $X_{\mathrm{c}} \simeq 55 \%$ and a hitherto unreported ordered phase in solid deuterium for $X<X_{\mathrm{c}}$.

The nature of the ordering in the new phase has been determined by analysing the NMR line shapes. There is a large distribution of local orientational order parameters for $X<X_{\mathrm{c}}$ and we interpret the transitions observed for low concentrations as a quadrupolar glass ordering of the orientational degrees of freedom of the $J=1$ molecules.
\end{abstract}

1. Introduction. - The purpose of this article is to report preliminary results for the observation of orientational ordering in solid deuterium for concentrations of the para species $(J=1)$ varying from 42 to $74 \%$. (For a review of the general properties of solid hydrogen and deuterium see reference [1]). The aim of these experiments was to explore the phase diagram for the orientational ordering in the vicinity of the critical concentration of para deuterium (approximately $55 \%$ ) below which previous studies [2] limited to high temperatures showed no evidence for ordered phases. Several experiments $[3,4,5,6,7]$ on solid hydrogen for low $J=1$ concentrations have shown that ordering does occur at low temperatures and the observed NMR line shapes show that there is a broad distribution of local orientational order parameters. These phases have been interpreted as a quadrupolar glass ordering of a random distribution of $J=1$ molecules similar to the spin glass ordering observed in dilute magnetic alloys. The nature of the transitions to the disordered phases in solid hydrogen seems to depend on the method of sample preparation : transi- tions over a broad temperature interval for samples condensed from the vapour phase onto a $10 \mathrm{~K}$ surface [6], as opposed to the much sharper transitions observed for samples obtained from a slow crystallisation of the liquid phase using fine copper wires to assure thermal contact $[4,5,7]$. Nevertheless, the details of the line shapes observed at the lowest temperatures are essentially identical and the conclusions drawn for the distribution of the order parameters remain true for both techniques.

In order to confirm the interpretation of the ordered phase in solid hydrogen for low $J=1$ concentrations we have sought a second example of a quadrupolar glass and toward this end we have investigated the temperature dependence of the NMR lineshapes in solid deuterium for a wide range of $J=1$ concentrations straddling the critical concentration.

2. Orientational order parameters and NMR spectra. - An array of molecular quadrupoles having orbital angular momentum $J=1$ can be described in a spin representation by five irreducible tensorial 
operators $\mathcal{O}_{i}^{m}(m=0, \pm 1, \pm 2)$ which are the operator equivalents of the spherical harmonics $Y_{2 \mathrm{i}}^{m}$ ( $\mathrm{i}$ is a site index). $\mathcal{O}_{\mathrm{i}}^{0}=\left(3 J_{Z}^{2}-2\right)_{\mathrm{i}} / \sqrt{6}$

$\mathcal{O}_{\mathrm{i}}^{ \pm 1}=\mp \frac{1}{2}\left(J_{Z} J_{ \pm}+J_{ \pm} J_{Z}\right)_{\mathrm{i}}$ and $\mathcal{O}_{\mathrm{i}}^{ \pm 2}=\frac{1}{2}\left(J_{ \pm}\right)_{\mathrm{i}}^{2}$.

These satisfy the orthonormality conditions $\operatorname{Tr}\left(\mathcal{O}^{m} \mathcal{O}^{n \dagger}\right)=\delta_{m, n}$.

The axes $(X, Y, Z)$ refer to an arbitrarily chosen laboratory frame. We can divide the five independent parameters needed to specify the orientational degrees of freedom of the quadrupoles into two classes; (i) the principal axes $\left(x_{\mathrm{i}}, y_{\mathrm{i}}, z_{\mathrm{i}}\right)$ of the tensorial operators $\mathcal{O}_{\mathbf{i}}^{m}$ for which the expectation values $\left\langle\mathcal{O}_{\mathbf{i}}^{ \pm 1}\right\rangle$ vanish and the $\left\langle\mathcal{O}_{i}^{ \pm 2}\right\rangle$ are real, and (ii) the intrinsic quadrupolar order parameters

$\sigma_{\mathrm{i}}=\sqrt{6}\left\langle\mathcal{O}_{\mathrm{i}}^{0}\right\rangle=\left\langle 3 J_{z_{\mathrm{i}}}^{2}-2\right\rangle$ and $\eta_{\mathrm{i}}=\left\langle J_{x_{\mathrm{i}}}^{2}-J_{y_{\mathrm{i}}}^{2}\right\rangle$.

If the system is in a pure state one can always choose the $z_{\mathrm{i}}$-axis of the local principal axes so that $\left\langle J_{x_{1}}\right\rangle=\left\langle J_{y_{i}}\right\rangle=0$; then either $\sigma_{i}=-2$ with $\eta_{\mathrm{i}}=0$, or $\sigma_{\mathrm{i}}=1$ with $\eta_{\mathrm{i}}= \pm \sqrt{1-\left\langle J_{z_{i}}\right\rangle^{2}}$.

However, if the or vitai angular momentum is quenched, i.e. $\left\langle\mathbf{J}_{\mathbf{i}}\right\rangle=0$, one can always choose the $z_{\mathrm{i}}$ axis so that $\sigma_{\mathrm{i}}=-2$ with $\eta_{\mathrm{i}}=0$ (for a pure state) a permutation of the labels $(x, y, z)$ of the principal axes transforms the values of the order parameters into $\sigma_{i}^{\prime}=1, \eta_{i}^{\prime}= \pm 1$ with respect to the permuted axes. The spin rotational coupling is comparable to the intramolecular nuclear dipole-dipole interactions and would lead to a much larger line width than that observed in the solid if the orbital momentum was not quenched. We therefore assume that quenching does occur in solid hydrogen and solid deuterium. (The dipole moments $\left\langle J_{x}\right\rangle,\left\langle J_{y}\right\rangle,\left\langle J_{z}\right\rangle$ must of course vanish if the molecular interactions leave the system time reversal invariant.) No values of $\sigma_{i}$ other than -2 and 1 are permitted for a pure state and it is therefore clear from the NMR results for solid hydrogen $[6,7]$ (which show that there is a broad distribution of different values of $\sigma_{i}$ ) that one cannot describe the system in terms of single particle wave functions. We therefore use the reduced single particle density matrix

$$
\rho_{\mathrm{i}}=\frac{1}{3} \mathbb{1}_{3}+\sum_{m=0, \pm 1, \pm 2} \mathcal{O}_{\mathrm{i}}^{m}\left\langle\mathcal{O}_{\mathrm{i}}^{m \dagger}\right\rangle
$$

to describe the orientational degrees of freedom $\nabla_{3}$ is the $3 \times 3$ unit matrix. As stated above, for the five parameters $\left\langle\mathcal{O}_{\mathrm{i}}^{m}\right\rangle$ needed to specify $\rho_{\mathrm{i}}$ we choose local axes $\left(x_{\mathrm{i}}, y_{\mathrm{i}}, z_{\mathrm{i}}\right)$ for which $\left\langle\mathcal{O}_{\mathrm{i}}^{ \pm 1}\right\rangle=0$ and $\left\langle\mathcal{O}_{i}^{ \pm 2}\right\rangle$ are real and the intrinsic local quadrupolar order parameters $\sigma_{\mathrm{i}}, \eta_{\mathrm{i}}$ (see reference [7]).

Given these order parameters one can determine the NMR spectra by expressing the intramolecular nuclear dipole-dipole and nuclear electric quadrupole interactions as products of the molecular tensorial operators $\mathcal{O}_{i}^{m}$ and nuclear spin operators and calculate the perturbation of the nuclear Zeeman levels for the case of a large applied magnetic field. In the ordered phases the intramolecular interactions do not average to zero and each para molecule $(I=1, J=1)$ contributes two satellites to the spectra with frequency separation

$\Delta v_{\mathrm{i}}=\frac{3}{2} d \cdot\left[\sigma_{\mathrm{i}} \cdot\left(\frac{3 \cos ^{2} \theta_{\mathrm{i}}-1}{2}\right)+\frac{3}{2} \eta_{\mathrm{i}} \sin ^{2} \theta_{\mathrm{i}} \cos 2 \varphi_{\mathrm{i}}\right]$

$d=25.24 \mathrm{kHz}$ is the sum of a dipolar term $\left(d_{\mathrm{m}}=2.74 \mathrm{kHz}\right)$ and a nuclear quadrupolar term $\left(d_{\mathrm{Q}}=22.50 \mathrm{kHz}\right)$ [8]. $\theta_{\mathrm{i}}, \varphi_{\mathrm{i}}$ are the polar angles specifying the orientation of the magnetic field with respect to the local axis $z_{\mathrm{i}}$.

In the long range ordered phase the order parameters take the same value at each site; $\sigma=-2$
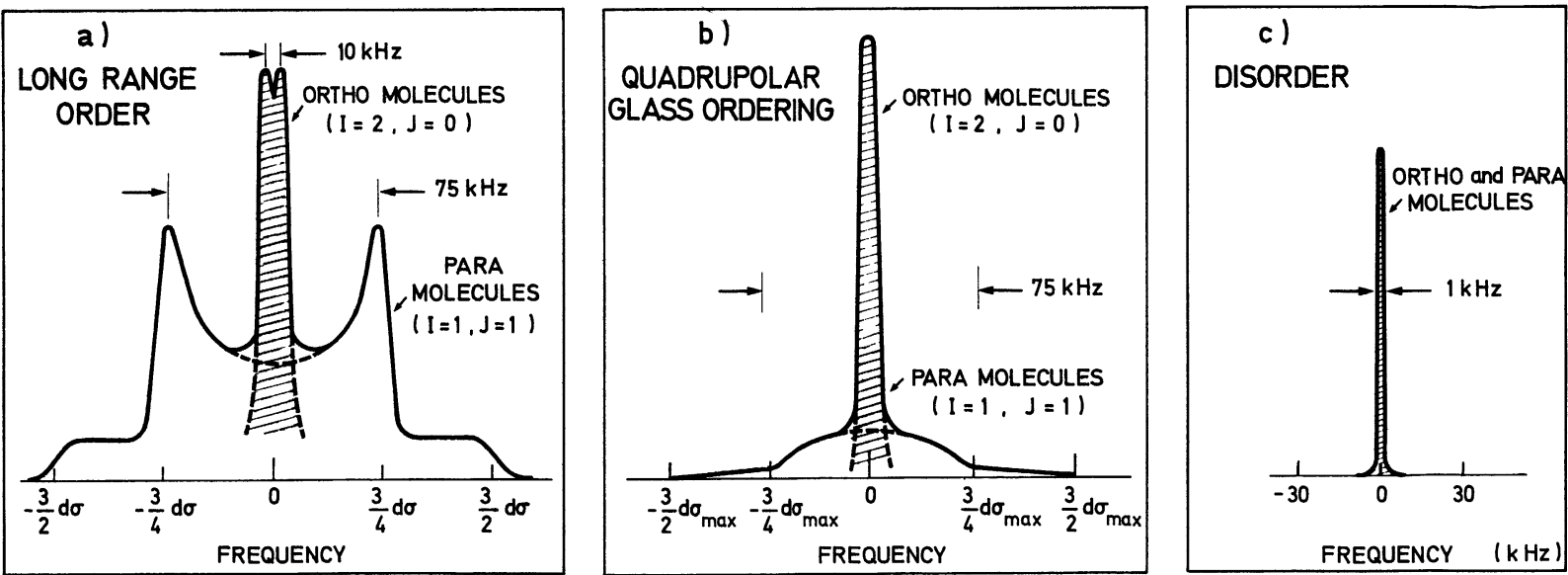

Fig. 1. - Predicted NMR line shapes for solid deuterium. a) Long range order. $\sigma=$ constant, $\eta=0$ (4 sublattice structure with local axes parallel to the body diagonals of the $\mathrm{Pa}_{3}$ structure [1]). The weak shoulders extending out to $v= \pm \frac{3}{2} d$ are observed only for para concentrations $X \gtrsim 80 \%$ b) Quadrupolar glass ordering. Broad distribution of local order parameters; $P(\sigma)=$ constant for $-\sigma_{\max }<\sigma<\frac{1}{2} \sigma_{\max }$, $\eta=0$. The relative intensity of the $I=1$ and $I=2$ components corresponds to a $J=1$ concentration of $50 \%$ and illustrates the relative weakness of the $I=1$ spectrum which contains the information on the orientational order parameters. c) Disorder phase. $T \gg T_{\mathrm{c}}(X)$. $\sigma=\eta=0$. 
(apart from corrections due to zero point effects and admixtures of higher $J$ states in the ground state) and $\eta=0$ (axial symmetry). Averaging over the angle $\theta_{\mathrm{i}}$ for a polycrystalline sample leads to a Pake doublet line shape for the contribution from the para molecules. In contrast to the case of solid hydrogen the $J=0$ molecules have nuclear spin $I=2$ and they contribute a narrow central line to the NMR spectrum which is also split due to the polarization of the ortho molecules $(J=0$ plus an admixture of $J=2$ states) by the para species [2]. The resulting line shape is shown in figure $1 a$.

For a quadrupolar glass ordering with a broad distribution $P(\sigma)$ of local order parameters $(P(\sigma)=$ constant for $\left.-\sigma_{\max }<\sigma<\frac{1}{2} \sigma_{\max }\right)$ the expected spectrum for the $I=1$ spins is a broad bell shaped line for $-\frac{3}{4} \mathrm{~d} \sigma_{\max }<v<\frac{3}{4} \mathrm{~d} \sigma_{\max }$ with weak shoulders extending out to $v= \pm \frac{3}{2} \mathrm{~d} \sigma_{\max }$. This is shown in figure $1 b$ together with the $I=2$ spectrum. The ratio of the area of the $I=1$ component to that of the $I=2$ component is $2 X / 5(1-X)$ where $X$ is the para concentration and in view of the large difference in the linewidths, $75 \mathrm{kHz}$ and $8 \mathrm{kHz}$ respectively, the spectrum of the quadrupolar glass phase is dominated by $I=2$ component. The scale of the NMR linewidth in solid deuterium is determined by the nuclear electric quadrupole interactions which is an order of magnitude larger than the intramolecular nuclear dipole interactions. The dipolar interactions alone determine the linewidths in solid hydrogen and consequently the intensity of the $I=1$ spectrum in solid deuterium is relatively very much weaker than that in solid hydrogen for the ordered phases. The observation of the $\mathrm{CW}$ absorption signal is also hampered by the long relaxation times [9]. For this reason we have investigated the NMR absorption spectrum for a sample having on initial para concentration of $74 \%$ for which the $I=1$ spectrum can be observed without undue difficulty, and the evolution of the spectra was followed as the sample aged.

EXPER IMENTAL RESUlTS. - Deuterium gas samples enriched in $J=1$ species (obtained by selective absorption on an alumina column [10]) were condensed into a Kel-F cell containing a large number of fine copper wires which were soldered to the mixing chamber of a dilution refrigerator. The NMR spectra were recorded using a low rf level Q-meter detector operating at $25 \mathrm{MHz}$ and signal averaging techniques were employed to improve the signal to noise ratios (see reference [7] for further details).

The initial $J=1$ concentrations were determined from thermal conductivity measurements of the gas phase at $77 \mathrm{~K}$ using a thin film thermal conductivity gauge [11]. Subsequent concentrations were inferred from the age of the samples by measuring the concentration of a twin sample aged at $4 \mathrm{~K}$ in a separate dewar. The para $\rightarrow$ ortho conversion rate determined by this method is in good agreement with theoretical predictions [12]. We found a decay rate for the para concentration $X$ given by $\dot{X}=-k^{\prime} X+k X^{2}$ with $k^{\prime}=(1.78 \pm 0.02) \times 10^{-3}$ per hour and $k=(0.55 \pm 0.02) \times 10^{-3}$ per hour. (Measurements of the conversion rate for $0.4<X<0.75$ do not seem to have been reported in the literature.)

The NMR sample was evaporated occasionally to check that there were no substantial variations in the concentrations of the two samples during the 34 days for which the samples were aged.
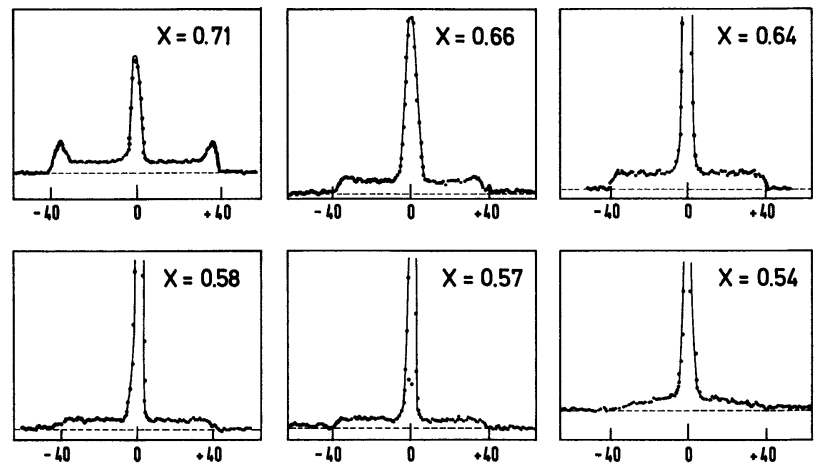

Fig. 2. - NMR absorption spectra recorded at $T \lesssim 50 \mathrm{mK}$ for different $J=1$ concentrations. The horizontal frequency scale is in $\mathrm{kHz}$. (The vertical scales are arbitrary.)

Typical absorption spectra recorded at temperatures much lower than the transition temperatures $T_{\mathrm{c}}(X)$ are shown in figure 2. At high para $(J=1)$ concentrations, the NMR spectra consist of approximately rectangular line shapes attributed to the $J=1$ species and a narrow central line due to the $J=0$ molecules. The rectangular line shapes are characteristic of the NMR spectra for the long range ordered phases for $-\frac{3}{2} d<v<\frac{3}{2} d$ allowing for small variation $(\sim 5 \%)$ in the order parameters. The peaks at the shoulders were observed only for $J=1$ concentrations greater than $70 \%$ and the details for $\frac{3}{2} d<v<3 d$ were below the noise level in the present experiments. The separation of the shoulders shown in figure 3 (taken from the maximum slopes of the absorption spectra) are well described by the order parameter

$\sigma=-2 \xi\left[1+0.066 T_{\mathrm{c}}(X) / T_{\mathrm{c}}(1)-0.021 T_{\mathrm{c}}(1) / T_{\mathrm{c}}(X)\right]$

$T_{\mathrm{c}}(X)$ are the experimental transition temperatures.

$\xi=0.98$ is a renormalisation factor $[2,8,13,14]$, the first term in the brackets accounts for the admixture of $J=3$ states and the second term accounts for the reduction of the order parameter due to zero point effects $[2,13,14,15]$. The results reported in figure 3 are in good agreement with those published by Meyer et al. for $X>59 \%$ [2].

For concentrations near the critical concentration $X_{\mathrm{c}} \approx 55 \%$, the form of the NMR spectra changed rapidly from a rectangular to a triangular line shape as predicted for the $I=1$ spectrum (Fig. 1b) for a 


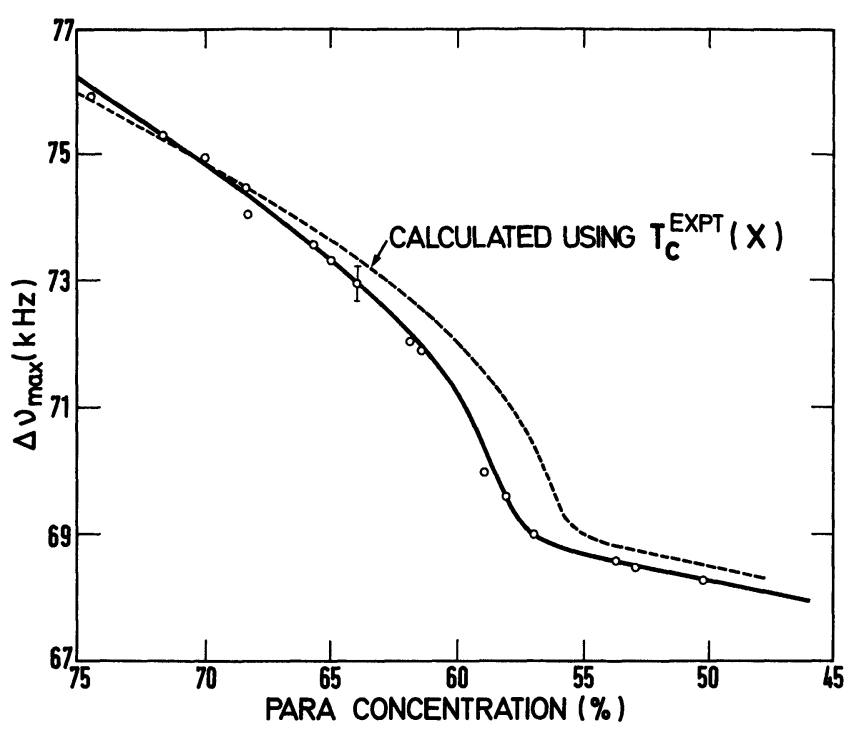

Fig. 3. - Concentration dependence of the frequency separation of the points of maximum slope $\left(\Delta v_{\max }=\frac{3}{2} \mathrm{~d} \sigma_{\max }\right)$ of the absorption spectra. The solid line is a guide to the eye. The broken line is calculated for

$$
\left|\sigma_{\max }\right| / 2=\xi\left[1+0.066 T_{\mathrm{c}}(X) / T_{\mathrm{c}}(1)-0.021 T_{\mathrm{c}}(1) / T_{\mathrm{c}}(X)\right]
$$

with $\xi=0.98 . T_{\mathrm{c}}(X)$ is the observed transition temperature. The solid circles refer to the present work after corrections for intermolecular dipolar broadening have been made [15].

quadrupolar glass ordering i.e. a broad distribution of local orientational order parameters. The signal to noise ratios are weak, excluding any quantitative comparison with the predicted spectra and we were unable to observe the triangular line shape for para concentrations below $42 \%$ using the present techniques.

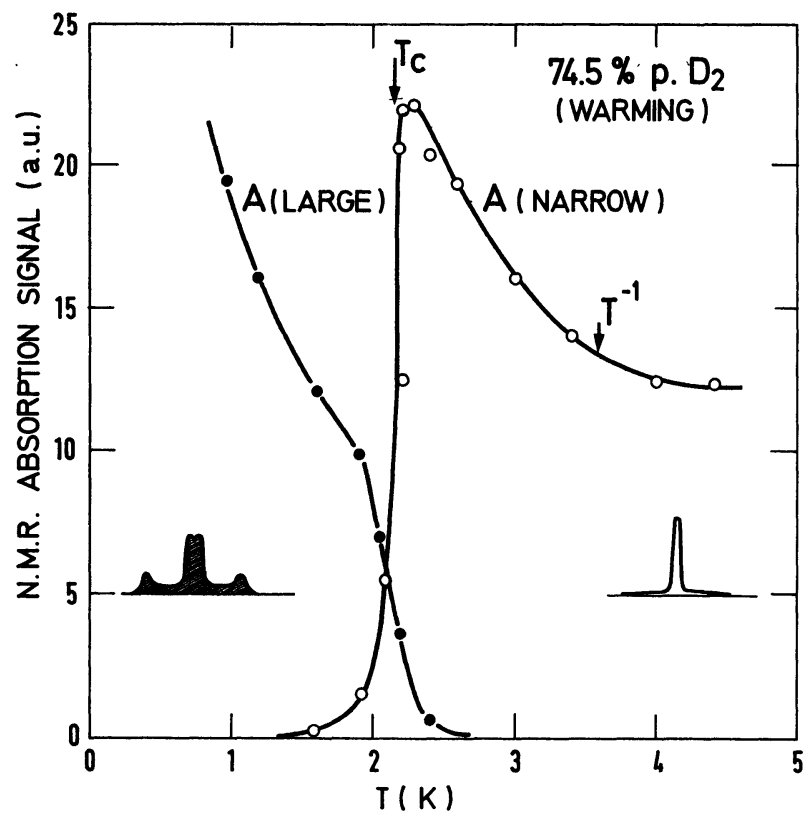

Fig. 4. - Example of the phase transition observed for a $J=1$ concentration of $74.5 \%$ showing the evolution of the areas of (i) the narrow line shape (disorder) and (ii) the broad line shape (orientational order) as the temperature is raised.
On warming the broad line shapes varied little until a critical temperature was reached where the lines changed rapidly over a narrow temperature interval to the narrow line shapes characteristic of the disordered phase. An example of this phase transition is shown in figure 4. The phase diagram for the transition temperatures $T_{\mathrm{c}}(X)$ as a function of the $J=1$ concentration $X$ is given in figure 5. The NMR results enable us to divide the phase diagram into three regions : a rotationally disordered phase, a long range orientationally ordered phase and a quadrupolar glass phase. Two different types of transitions are observed : transitions to the disordered phase on warming (from either the long range ordered phase or the glass phase), and transitions to the glass phase from the long range ordered phase by aging at low temperatures. The transitions on warming represented by the solid line in figure 5 occur over a narrow temperature interval and are in reasonably good agreement with those reported elsewhere [16] for $X>55 \%$.

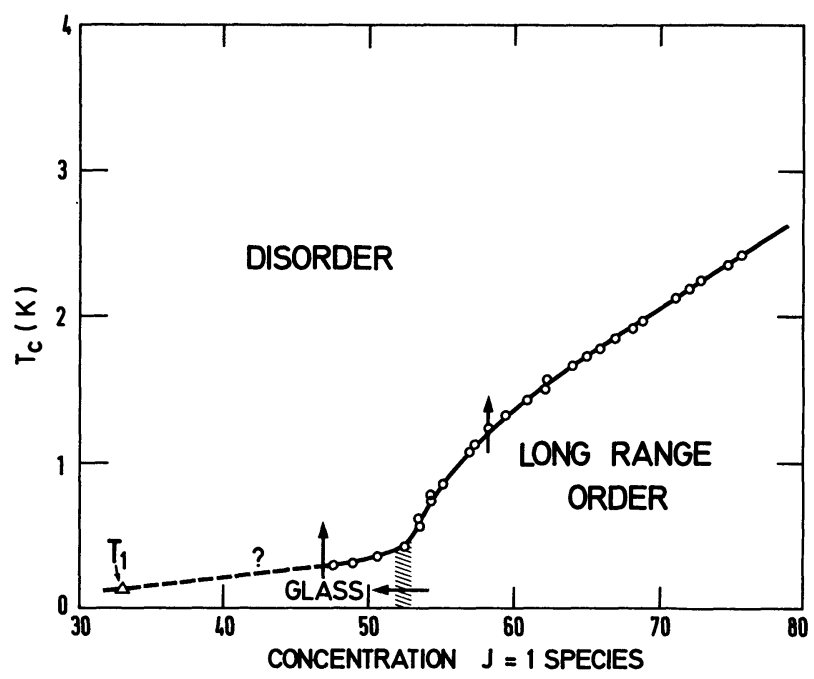

Fig. 5. - Phase diagram for solid deuterium determined from the changes in the NMR spectra on either warming (vertical arrows) or aging (horizontal arrow). The solid circles refer to the transitions on warming. (The transitions on cooling occur at lower temperatures and are not as reproducible as the transitions on warming - this hysteresis diminishes with thermal cycling. The triangle refers to measurements of the relaxation time [9].)

There is a distinct change in the concentration dependence of the critical temperatures $T_{\mathrm{c}}(X)$ for concentrations lower than the critical concentration $X_{\mathrm{c}} \simeq 55 \%$, and analogous to the observations in solid hydrogen we find

$$
T_{\mathrm{c}}^{\mathrm{D}_{2}} \simeq(0.4 \pm 0.1) X^{1 / 2} \mathrm{~K} \text { for } X<X_{\mathrm{c}} \simeq 55 \% \text {. }
$$

Although, as a result of signal to noise problems, we have not been able to determine $T_{\mathrm{c}}(X)$ very reliably for $X<X_{\mathrm{c}}$, the values of $T_{\mathrm{c}}^{\mathrm{D}_{2}}$ are significantly smaller than expected in comparison with the values observed for hydrogen $[6,7]$,

$T_{\mathrm{c}}^{\mathrm{H}_{2}}=0.55(X-0.10)^{1 / 2} \mathrm{~K}$ for $X<X_{\mathrm{c}} \simeq 59 \%$. 
The electrostatic quadrupole-quadrupole interaction constant for deuterium $\left(\Gamma_{\mathrm{D}}=1.07 \mathrm{~K}\right)$ is larger than that for hydrogen $\left(\Gamma_{\mathbf{H}}=0.84 \mathrm{~K}\right)$ and taking the results obtained for hydrogen one would expect transition temperatures

$$
T_{\mathrm{c}}^{\mathrm{D}_{2}} \approx\left(\Gamma_{\mathrm{D}} / \Gamma_{\mathrm{H}}\right) T_{\mathrm{c}}^{\mathrm{H}_{2}} \approx 0.7 X^{1 / 2} \mathrm{~K}>T_{\mathrm{c}}^{\mathrm{H}_{2}}
$$

if short range electrostatic quadrupole-quadrupole interactions were alone responsible for ordering in the glass phase.

On the other hand, the critical temperatures for pure orthohydrogen and pure paradeuterium do scale according to the quadrupole interaction constants $\Gamma$ which is consistent with the view that the long range ordering at high $J=1$ concentrations is determined almost completely by the quadrupole-quadrupole interactions. Weak long range $\left(R^{-3}\right)$ anisotropic interactions due to the coupling of the rotational motion with the phonon system are present but current estimates of the strengths of these interactions are too small to associate the quadrupolar glass ordering with the indirect interactions alone. Harris [13] finds $V_{\text {ind. }} \lesssim 0.02 \mathrm{~K}$ while Van Kranendonk and Sears [17] give $V_{\text {ind. }} \lesssim 0.09 \mathrm{~K}$. It is interesting to note however, that $V_{\text {ind. }} \propto\left(M \omega_{\mathrm{D}}^{2} R_{0}^{2}\right)^{-1}$ where $M$ is the molecular mass, $\omega_{\mathrm{D}}$ the Debye frequency and $R_{0}$ the lattice separation [13] and $V_{\text {ind. }}^{\mathrm{D}_{2}} / V_{\text {ind. }}^{\mathrm{H}_{2}} \simeq 0.78$. This scaling is consistent with the ratios of the transition temperatures observed in $\mathrm{D}_{2}$ and $\mathrm{H}_{2}$ for $X<X_{\mathrm{c}}$. Calculations of the indirect interactions are not straightforward and it would be useful to determine the phase diagram at high densities and low temperature to test the nature of the interactions at low concentrations. The transition temperatures at high concentrations due to quadrupolar interactions $\left(R^{-5}\right)$ would increase much more rapidly with density than would transition temperatures attributable to the indirect interactions $\left(R^{-3}\right)$.

Although we have not been able to observe the $I=1$ spectrum for $X<42 \%$ using CW NMR techniques, measurements of the nuclear spin relaxation times [9] for $X=33 \%$ have shown evidence for a rapid freezing of the orientational degrees of freedom at $T=160 \pm 20 \mathrm{mK}$. This point has been included in the phase diagram (Fig. 5) and further measurements of this nature are needed to complete the phase diagram for $X<42 \%$.
The transitions from the long range ordered phase to the glass represented by the hatched region in figure 5 have been determined from the evolution of the low temperature line shapes shown in figure 2 as the sample is allowed to age. Although the evolution of the rectangular line shape to the triangular line shape occurs smoothly over a concentration range of approximately $10 \%$ near the critical concentration, the concentration dependence of the frequency separation of the shoulders, $\Delta v_{\max }=\frac{3}{2} \mathrm{~d} \sigma_{\max }$ (Fig. 3) changes much more rapidly at the critical concentration. This is interpreted as a transition from long range order for $X>X_{\mathrm{c}}$ to a quadrupolar glass phase for $X<X_{\mathrm{c}}$. For $X<X_{\mathrm{c}}$ it is impossible to realize an infinite cluster of $J=1$ molecules whose orientations are correlated according to the four sublattice $\mathrm{Pa}_{3}$ structure.

3. Conclusion. - NMR techniques provide a powerful tool for studying the orientational degrees of freedom in solid deuterium. The NMR spectra are directly related to the orientational order parameters and the NMR line shapes can be used to deduce the distribution of local order parameters for different concentrations of the $J=1$ species. The preliminary experimental results reported here show that for $J=1$ concentrations below a critical concentration of approximately $55 \%$, there is a broad distribution of local order parameters revealing the existence of a hitherto unreported quadrupolar glass phase in solid deuterium. The transitions from the glass phase to the disordered phase occur over a narrow temperature interval and the concentration dependence of the transition temperatures is qualitatively similar to that observed in solid hydrogen.

The present experiments have not permitted the observation of the glass phase for $X<42 \%$ and we plan to extend these measurements to lower concentrations using improved $\mathrm{CW}$ techniques to obtain better signal to noise ratios and by measuring the nuclear spin lattice relaxation times.

Acknowledgments. - Numerous discussions with A. Abragam, M. Goldman, D. Esteve and C. Urbina are gratèfully acknowledged.

\section{References}

[1] Silvera, I. F., in Low Temp. Phys. LT14, 5 p. 123. Proc. 14th Int. Conf. on Low Temp. Phys., Helsinki, 1975 (North Holland Publishing, Amsterdam)

[2] Meyer, H., Weinhaus, F., Maraviglia, B., Phys. Rev. 6 (1972) 1112 (and reference therein).

[3] Sullivan, N. S. and Pound, R. V., Phys. Lett. 39A (1972) 23.

[4] Ishimito, H., Nagamine, K. and Kimura, Y., J. Phys. Soc. Japan 35 (1973) 300.

[5] Husa, D. L. and Daunt, J. G., Phys. Lett. A 65 (1978) 354.
[6] Vinggar, H. J., Byleckie, J. J. and Pound, R. V., Phys. Rev. B 16 (1977) 3016.

[7] Sullivan, N. S., Devoret, M., Cowan, B. P. and Urbina, C., Phys. Rev. B 17 (1978) 5016.

[8] Hardy, W. N. and Berlinsky, A. J., Phys. Rev. B 8 (1973) 4996.

[9] Sullivan, N. S. and Devoret, M., J. Physique 39 (1978) C6-92.

[10] Depate, D. A. and Mills, R. L., Rev. Sci. Instr. 39 (1968) 105. 
[11] Devoret, M., Sullivan, N. S. and Deschamps, P. (to be published).

[12] Motizuki, K., J. Phys. Soc. Japan 17 (1962) 1192.

[13] Harris, A. B., Phys. Rev. B 1 (1970) 1881.

[14] Sullivan, N. S., Vinegar, H. J. and Pound, R. V., Phys. Rev. B 12 (1975) 2596.
[15] Pedroni, P. L., Schweitzer, R. and Meyer, H., Phys. Rev. B 14 (1976) 896.

[16] Ramm, D., Meyer, H. and Mills, R. M., Phys. Rev. 11 (1970) 2763.

[17] Van Kranendonk, J. and Sears, V. F., Can. J. Phys. 44 (1966) 313. 